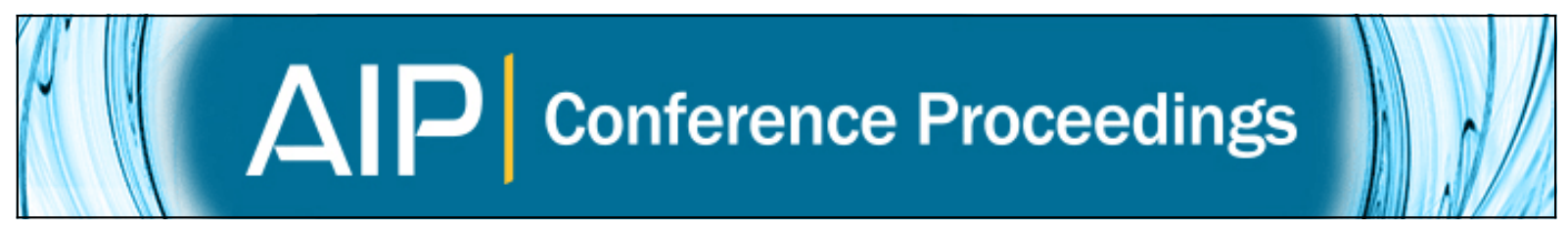

Volumetric Fraction Dynamic Measurement in Oil-Water-Gas Multiphase Horizontal Pipe Flow with Dual Energy Gamma-Ray

Donghui Li, Yingxiang Wu, Keren Wang, and Xingfu Zhong

Citation: AIP Conference Proceedings 914, 409 (2007); doi: 10.1063/1.2747460

View online: http://dx.doi.org/10.1063/1.2747460

View Table of Contents: http://scitation.aip.org/content/aip/proceeding/aipcp/914?ver=pdfcov

Published by the AIP Publishing

Articles you may be interested in

Multiphase Flow Measurement System of Oil Well

AIP Conf. Proc. 914, 625 (2007); 10.1063/1.2747490

Effect of Slug Flow on Pipe Vibration in Horizontal Pipeline

AIP Conf. Proc. 914, 537 (2007); 10.1063/1.2747477

Measurement Of Multiphase Flow Water Fraction And Water-cut

AIP Conf. Proc. 914, 232 (2007); 10.1063/1.2747436

Electronics Design for Dual Energy Gamma-Ray Multiphase Flow Meter

AIP Conf. Proc. 914, 172 (2007); 10.1063/1.2747427

Multiphase Flow Measurement by Dual Gamma Ray Tomography

AIP Conf. Proc. 914, 31 (2007); 10.1063/1.2747410 


\title{
Volumetric Fraction Dynamic Measurement in Oil-Water- Gas Multiphase Horizontal Pipe Flow with Dual Energy Gamma-Ray
}

\author{
Donghui Li, Yingxiang $\mathrm{Wu}$, Keren Wang and Xingfu Zhong \\ Institute of Mechanics, Chinese Academy of Sciences
}

\begin{abstract}
The problems of how to measuring the volumetric fractions of oil-water-gas multiphase flow are still a problem remaining to be solved in oil industry. With the technological development of nuclear radioactive inspection, dual-energy $\gamma$-ray techniques make it possible to investigate the concentration of the different components on the crosssection of oil-water-gas multiphase pipe-flow. The dual-energy Gamma-ray technique is based on the materials attenuation coefficients measurement. It is comprised of two radioactive isotopes of ${ }^{241} \mathrm{Am}$ and ${ }^{137} \mathrm{Cs}$ with emission energies of $59.5 \mathrm{keV}$ and $662 \mathrm{keV}$. In order to measuring the material's attenuation dose rate, some nuclear instruments and data acquisition system were designed; a number of static and dynamic tests were carried out in the Multiphase Laboratory, Institute of Mechanics, Chinese Academy of Sciences. The oil-water-gas three phases of medium have been investigated to simulate different media volumetric fraction distributions on the experimental flow loop. The measurement results of attenuation intensities were obtained; the linear attenuation coefficients and the volumetric fractions were studied and measurement error was discussed in this paper as well.
\end{abstract}

Keywords: Volumetric fraction, Multiphase flow, Dual-energy $\gamma$-ray

\section{INTRODUCTION}

Oil-water-gas multiphase mixed transportation in oil pipes is extensively used in ocean oil industries. How to metering the volumetric fractions of oil-water-gas multiphase flow is important in oil industry. Related research work started since the 1980s. The measurement of component ratios in multiphase flows using $\gamma$ ray attenuation was first suggested by Abouelwafa and Kendall (1980) [1], and the technique has been used in many current commercial multiphase metering systems. Single energy $\gamma$ ray technique working as a densimeter is satisfactory in two components measurement of gas/liquid or oil/water pipe-flow if not considering the concentration distribution of the different components in the cross-section. But in oil-water-gas three phase flow, the situation is different. Dual energy $\gamma$ ray techniques have been developed rapidly in the last decade, the different attenuation properties of the three phase's media in the oil pipe are used to obtain the components fraction information. Dual energy $\gamma$ ray technique has been applied to a case with three basis materials having distribution of linear attenuation coefficients with an obvious variance. It is proved to be a very promising technique for the purpose of simple and fast estimating the volumetric fractions of oil-water-gas multiphase flow, and it becomes the constituent part of radioactive based multiphase meter or tomography [2]. 


\section{THEORIES}

\section{Dual Energies Theories}

The following absorption law describes the mathematical relation between the $\gamma$ ray intensity $I_{0}$ radiated by the $\gamma$ ray source and the remaining intensity $I$ after passing through a massive object with a given length $L$ and a density $\rho$ (Minder and Liechti 1955[3]; Morneburg 1995[4]):

$$
I=I_{0} e^{-\eta(Z E) \rho L}
$$

The absorption coefficient $\eta$ is a function of the $\gamma$ ray energy $E$ and the atom number $Z$.

Under the $\gamma$ ray energy $E$, then:

$$
I(E)=I_{0}(E) e^{-\mu(E) L}
$$

For an oil-water-gas three-phase flow, the attenuation coefficient of the mixture $\mu(E)$ is represented by

$$
\mu(E)=\alpha \mu(E)_{o}+\beta \mu(E)_{w}+\gamma \mu(E)_{g}
$$

where $\mu(E)_{o}, \mu(E)_{w}$ and $\mu(E)_{g}$ are the linear attenuation coefficients of the oil, water and gas and $\alpha, \beta, \gamma$ are the respective volumetric fractions. The transmitted intensity $I$ through a thickness $L$ of an oil/water/gas mixture is therefore

$$
I_{0} e^{-\left(\alpha \mu(E)_{o}+\beta \mu(E)_{w}+\gamma \mu(E)_{g}\right) L}
$$

The build-up factor can be eliminated through a good collimation at the source and detector. If the transmitted fluxes $I_{1}$ and $I_{2}$ at two energies $E_{1}$ and $E_{2}$ are measured, the volume fractions can be calculated from the linear attenuation coefficients of the flow components at $E_{1}$ and $E_{2}$

$$
\begin{aligned}
& \ln \left(\frac{I}{I_{0}}\right)_{1}=-\left[\alpha \mu\left(E_{1}\right)_{o}+\beta \mu\left(E_{1}\right)_{w}+\gamma \mu\left(E_{1}\right)_{g}\right] L \\
& \ln \left(\frac{I}{I_{0}}\right)_{2}=-\left[\alpha \mu\left(E_{2}\right)_{o}+\beta \mu\left(E_{2}\right)_{w}+\gamma \mu\left(E_{2}\right)_{g}\right] L \\
& \alpha+\beta+\gamma=1
\end{aligned}
$$

There are three equations with three unknown volumetric fractions $\alpha, \beta$ and $\gamma$. In practice, $\mu\left(E_{1}\right)_{g}$ and $\mu\left(E_{2}\right)_{g}$ can be taken as zero without appreciable error in the volume fraction measurements because of the low density of the gas phase.

The linear attenuation coefficients of water and mineral oil (kerosene) over the same energy range reveal differences in the photon absorption, which may be used to distinguish the two materials. Fig.1 shows that the photon attenuation is greater in water than in oil. This is because the oxygen has a higher atomic number than the carbon, and also because water $\left(\rho=1.00 \mathrm{gcm}^{-3}\right)$ has a higher density than most mineral oils (typically $\rho=$ $0.80 \sim 0.90 \mathrm{gcm}^{-3}$ ).

Since the photoelectric interaction is a stronger function of photon energy and atomic number than the Compton interaction, one sees a great contrast in the linear attenuation coefficients of oil and water in the region where the photoelectric effect dominates the interaction cross-section of both materials [5].This is illustrated in Fig.2, where the relative difference in the linear attenuation coefficient is plotted as a function of photon energy for the same energy interval. It indicates that the $\gamma$ ray measurement system relying on the photon attenuation in oil and water to distinguish the two materials can obtain best results in the energy region below $40 \mathrm{keV}$.

In this paper, the $\gamma$ ray system is comprised of two radioactive isotopes of ${ }^{241} \mathrm{Am}$ and ${ }^{137} \mathrm{Cs}$, which have emission energies of $59.5 \mathrm{keV}$ and $662 \mathrm{keV}$. The lower $\gamma$ ray energy is higher than $40 \mathrm{keV}$, so that the linear attenuation coefficients of two energies in oil and water are somewhat close than expected. The necessary condition for above equations to be linearly independent is: 


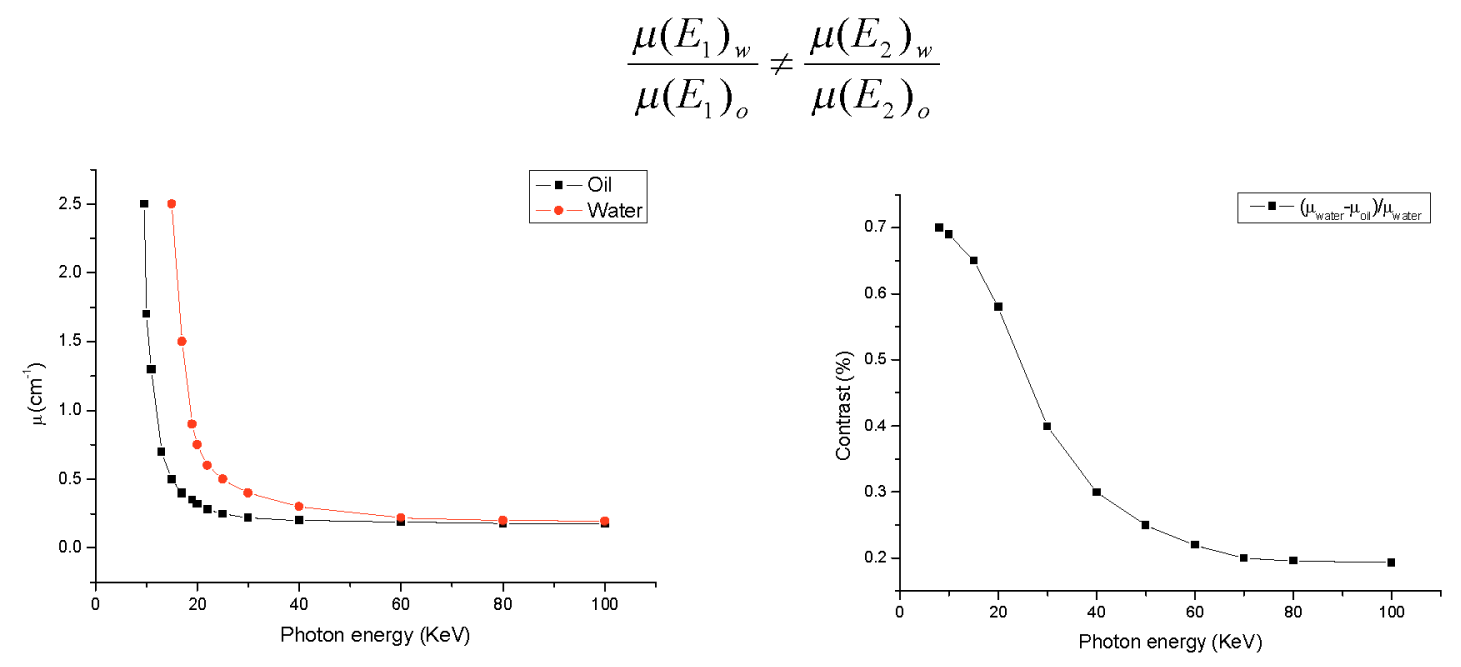

FIGURE 1. Water and oil attenuation coefficients

FIGURE 2. Radiographic contrast between oil and water

But the too close linear attenuation coefficients of two energies may propagate small errors in the measurements of the intensities into large errors in the estimation of the thickness; this situation is avoided only by a proper selection of the energies.

\section{Geometry Correction}

A single beam dual energy $\gamma$ ray was used as a densimeter in this measurement. The oil-water-gas three phase media have different densities, influenced by gravity, they stay in different layers in the horizontal pipe, and also have different dynamic distributions in the pipe. In other hand, because there are very complicated flow regimes in the oil-water-gas multiphase horizontal pipe flow, and the single beam $\gamma$ ray could not cover the whole pipe cross section, it only pass through the centre of the pipe, so the measured results must be flow regime dependent, and need a geometry correction.

The correction is based on a layered distribution of three phases in horizontal pipe, with water in the bottom layer, oil in the middle layer and gas in the top layer. Let $\alpha, \beta$ and $\gamma$ be the linear fractions of oil, water and gas, respectively, as shown in Fig.3. If $\mathrm{R}$ is the radius of the pipe, it can be shown that the half angle $\theta$ covered by the portion occupied by water will be:

$$
\theta=\arccos (1-\beta)
$$

Therefore, the total volume occupied by water is:

$$
V_{\text {water }}=\theta R^{2}-R^{2} \cdot \cos \theta \cdot \sin \theta=R^{2}(\theta-\cos \theta \cdot \sin \theta)
$$

So the corrected volumetric fraction of water becomes:

$\beta^{\prime}=\frac{\theta-\cos \theta \cdot \sin \theta}{\pi}$

Similarly, it is easy to get the corrected volumetric fractions of oil and gas, $\alpha^{\prime}$ and $\gamma^{\prime}$. In the following discussions, the superscript prime for the corrected volumetric fractions will be dropped.

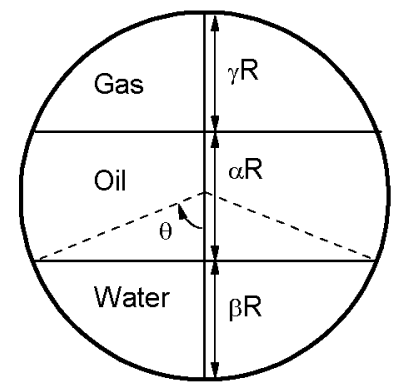

FIGURE 3. Oil-water-gas distributions. 


\section{EXPERIMENTAL SETUP}

\section{$\gamma$ Ray Source}

In this paper, the $\gamma$ ray system is comprised of two radioactive isotopes of ${ }^{241} \mathrm{Am}$ and ${ }^{137} \mathrm{Cs}$, which have emission energies of $59.5 \mathrm{keV}$ and $662 \mathrm{keV}$. Both of radioactive isotopes were assembled and shielded in a thick lead pot to prevent the harmful high energy emission of ${ }^{137} \mathrm{Cs}$. The radiation intensities of both isotopes are $100 \mathrm{mCi}$ and $20 \mathrm{mCi}$. The reason for choosing a greater radiation intensity for ${ }^{241} \mathrm{Am}$ than ${ }^{137} \mathrm{Cs}$ is that ${ }^{241} \mathrm{Am}$ has a lower photon energy therefore weakens in the penetration of the measurement pipe. A collimated single $\gamma$ ray beam of $20 \mathrm{~mm}$ in diameter comes out from the bottom of the source pot and can be turn on/off by a mechanical switch to ensure the operation safety.

\section{Scintillator Detector}

An important step in the dual energy $\gamma$ ray design is the selection of scintillation detectors. Two parameters are important while selecting the scintillation detectors: detection efficiency and decay constant of the scintillator. High detection efficiency is required to reduce the source strength and short decay constant is required for high count rate to avoid pulse pile up or saturation. NaI ( $\mathrm{Tl}$ ) crystal is most commonly used scintillator for its highest detection efficiency; it is very important for ${ }^{137} \mathrm{Cs}$ due to its high emission energy (662keV) and strong penetration ability.

A column crystal of Scintillator was made in size $40 \mathrm{~mm}(\mathrm{H}) \times 40 \mathrm{~mm}$ (D) and assembled with a photo multiplier tube (PMT). The total diameter of the detector is $\$ 55 \mathrm{~mm}$ and the length is $220 \mathrm{~mm}$. In addition, a collimation hole was designed of size of $30 \mathrm{~mm}(\mathrm{~W}) \times 50 \mathrm{~mm}(\mathrm{~L}) \times 150 \mathrm{~mm}(\mathrm{H})$, which was mounted on the top of detector.

\section{Nuclear Instrument}

The nuclear instrument presented in this project was designed as a multi-channels instrument. The system operated in counter mode. It is comprised of high voltage source, amplifier, shaping amplifier and programmable counter data acquisition system. The diagram is shown in Fig.4.

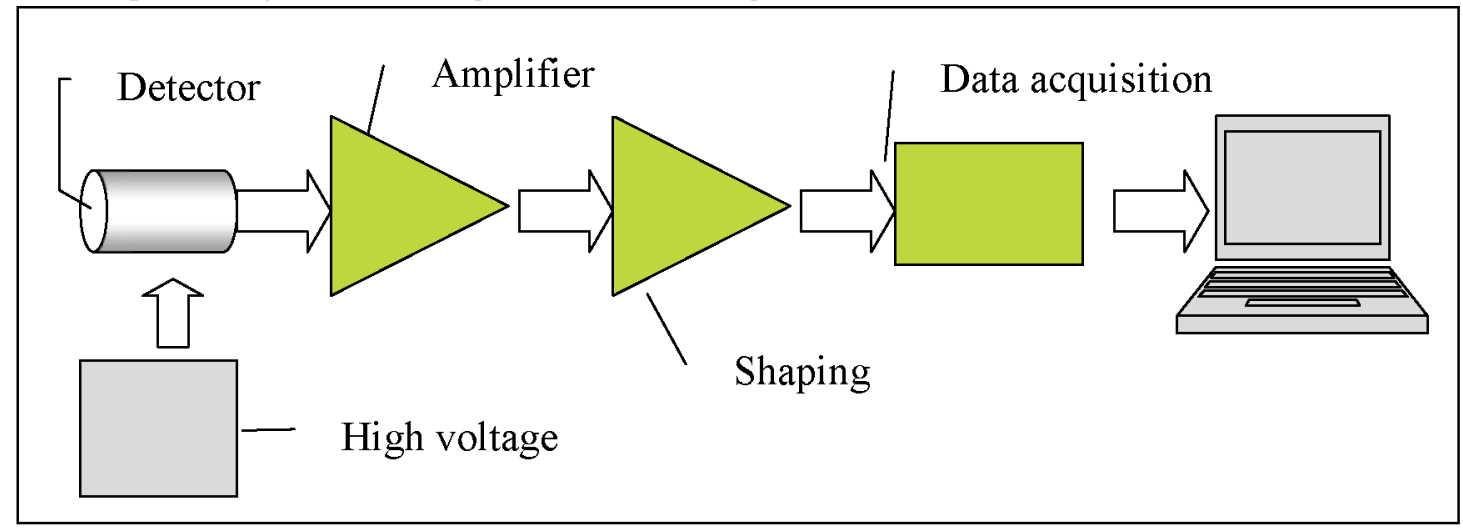

FIGURE 4. Nuclear instrument

\section{Flow Loop}

The flow loop is located in Multiphase Flow Laboratory, Institute of Mechanics. It is used to simulate oil-watergas three phase flow. The flow loop is shown in Fig.5. The experiment section is comprised of a 20 metre long horizontal pipe, including $3 \mathrm{~m}$ upstream pipe and $3 \mathrm{~m}$ downstream vertical pipe; the pipe diameter is $50 \mathrm{~mm}$ and made of transparent Plexiglass in order to observe the flow regime conveniently. 
Water from the water tank is pumped to the experiment pipe with a water pump, and passes through an electricalmagnetic flow meter for measuring the flow rate; oil in the oil tank is pumped and metered by an oil pump and oil flow rate meter; gas comes from air compressor and is metered by a gas mass flow meter. Three kinds of media are mixed in the ' $Y$ ' type entrance of the experiment pipe. In the end of the experiment pipe, the fluid passes through a ' $T$ ' junction pipe in order to pre-separate part of gas and oil, then the remaining medium passes in a two class gravity separation tank. After a period of stablizing time, the oil and water are well separated and pumped to the water tank and the oil tank ready for recycling.
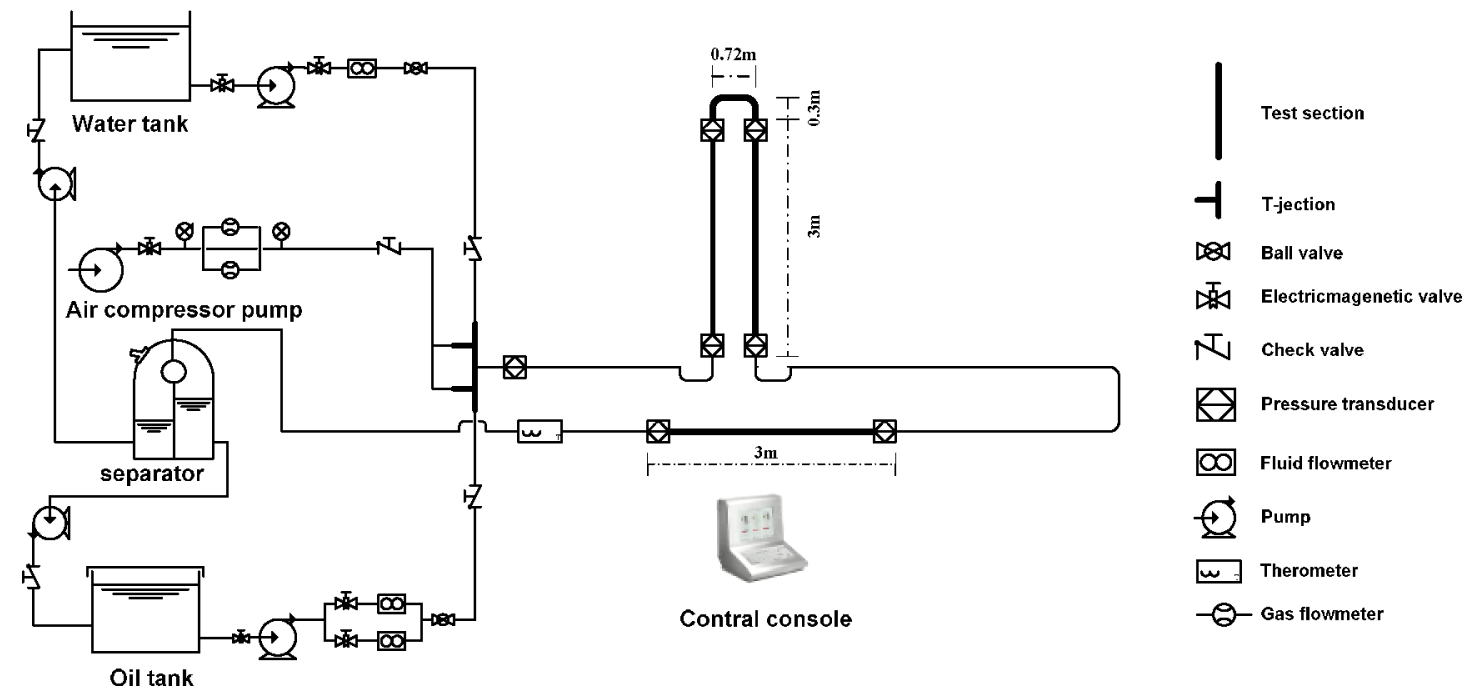

FIGURE 5. Multiphase flow loop

\section{Static Calibration Rack and Vessels}

Before the dynamic test, the dual energies $\gamma$ ray system has to be statically calibrated. An experimental rack and 4 square vessels were designed to be a static test rack, which stood separately from the multiphase flow loop; it also can work as a static calibration rack. The rack is one meter high and supports the source pot on its top. Plexiglass vessels were designed to contain oil-water-gas three phase media, in size of $100 \mathrm{~mm}(\mathrm{~L}) \times 100 \mathrm{~mm}(\mathrm{~W}) \times 600 \mathrm{~mm}(\mathrm{H})$ and with wall thickness of $5 \mathrm{~mm}$. The vessel works in horizontal direction with total thickness of $110 \mathrm{~mm}$ and a valid space of $100 \mathrm{~mm}$. Its position (height) between $\gamma$ ray source and detector could be adjusted by two jacks depending on the experiment requirements. The rack is shown in Fig.6.

\section{EXPERIMENTAL ARRANGEMENT}

The oil-water-gas three phase flow is very complicated and it is impossible to test all the flow rate combinations for each phase. In order to study the three phase materials attenuation character, an experimental arrangement was designed as shown in Table 1, including total 6 groups of measurements in the experiment. All the flow rate data are the superficial flow rate obtained from the flow meter of each phase.

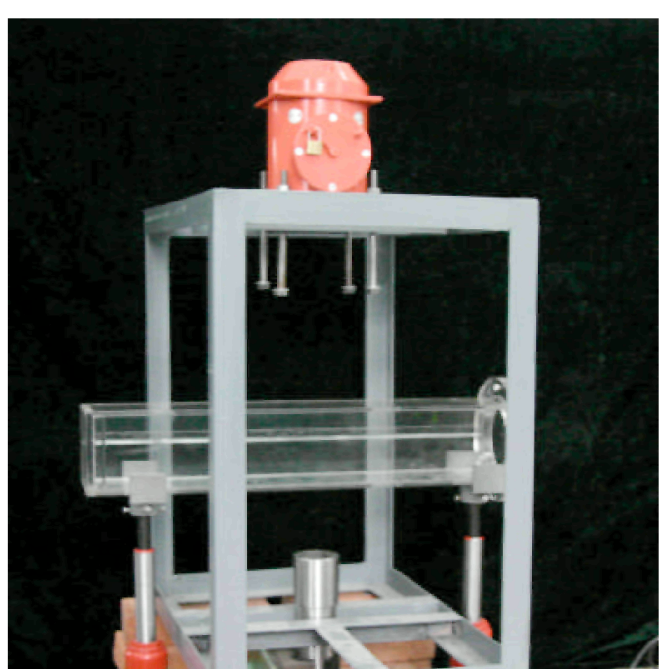

FIGURE 6. The static experimental rack 
TABLE. 1 ( Gas $1=20 \mathrm{SL} / \mathrm{min}$, Gas2 $=100 \mathrm{SL} / \mathrm{min}$ )

\begin{tabular}{ccccccccc}
\hline Oil & \multicolumn{7}{c}{ Water } \\
\hline $0.75 \mathrm{~m}^{3} / \mathrm{h}$ & $2.5 \mathrm{~m}^{3} / \mathrm{h}$ & $3.75 \mathrm{~m}^{3} / \mathrm{h}$ & $5 \mathrm{~m}^{3} / \mathrm{h}$ & $6.25 \mathrm{~m}^{3} / \mathrm{h}$ & $7.5 \mathrm{~m}^{3} / \mathrm{h}$ & $8.75 \mathrm{~m}^{3} / \mathrm{h}$ & $10 \mathrm{~m}^{3} / \mathrm{h}$ & $12.5 \mathrm{~m}^{3} / \mathrm{h}$ \\
$1.5 \mathrm{~m}^{3} / \mathrm{h}$ & $2.5 \mathrm{~m}^{3} / \mathrm{h}$ & $3.75 \mathrm{~m}^{3} / \mathrm{h}$ & $5 \mathrm{~m}^{3} / \mathrm{h}$ & $6.25 \mathrm{~m}^{3} / \mathrm{h}$ & $7.5 \mathrm{~m}^{3} / \mathrm{h}$ & $8.75 \mathrm{~m}^{3} / \mathrm{h}$ & $10 \mathrm{~m}^{3} / \mathrm{h}$ & $12.5 \mathrm{~m}^{3} / \mathrm{h}$ \\
$3.6 \mathrm{~m}^{3} / \mathrm{h}$ & $2.5 \mathrm{~m}^{3} / \mathrm{h}$ & $3.75 \mathrm{~m}^{3} / \mathrm{h}$ & $5 \mathrm{~m}^{3} / \mathrm{h}$ & $6.25 \mathrm{~m}^{3} / \mathrm{h}$ & $7.5 \mathrm{~m}^{3} / \mathrm{h}$ & $8.75 \mathrm{~m}^{3} / \mathrm{h}$ & $10 \mathrm{~m}^{3} / \mathrm{h}$ & $12.5 \mathrm{~m}^{3} / \mathrm{h}$ \\
\hline
\end{tabular}

\section{RESULTS AND DISCUSSIONS}

\section{Discussions on Measurement Accuracy}

With a well collimated scintillation detector and a dual energy $\gamma$ ray source, a series experiments were carried out on multiphase flow loop in IOM. The test curves on oil-water-gas three phase flow were obtained. A test curve of slug flow was shown in Fig.7. The dispersed areas are bubbles, the continuous area is the liquid phase, and the flow regime is shown on top diagram in Fig.7. The left diagram is the signal of ${ }^{241} \mathrm{Am}$; the right diagram comes from ${ }^{137} \mathrm{Cs}$ channel.

When the bubbles pass through the test section, the materials attenuation is reduced; then the count rate rises, when the liquid fills the whole test section of the pipe, the materials attenuation reaches it maximum, then the count rate drops down. In ${ }^{137} \mathrm{Cs}$ channel, the signal character is the same as ${ }^{241} \mathrm{Am}$ channel but its total attenuation is less because ${ }^{137} \mathrm{Cs}$ has higher emission energy.

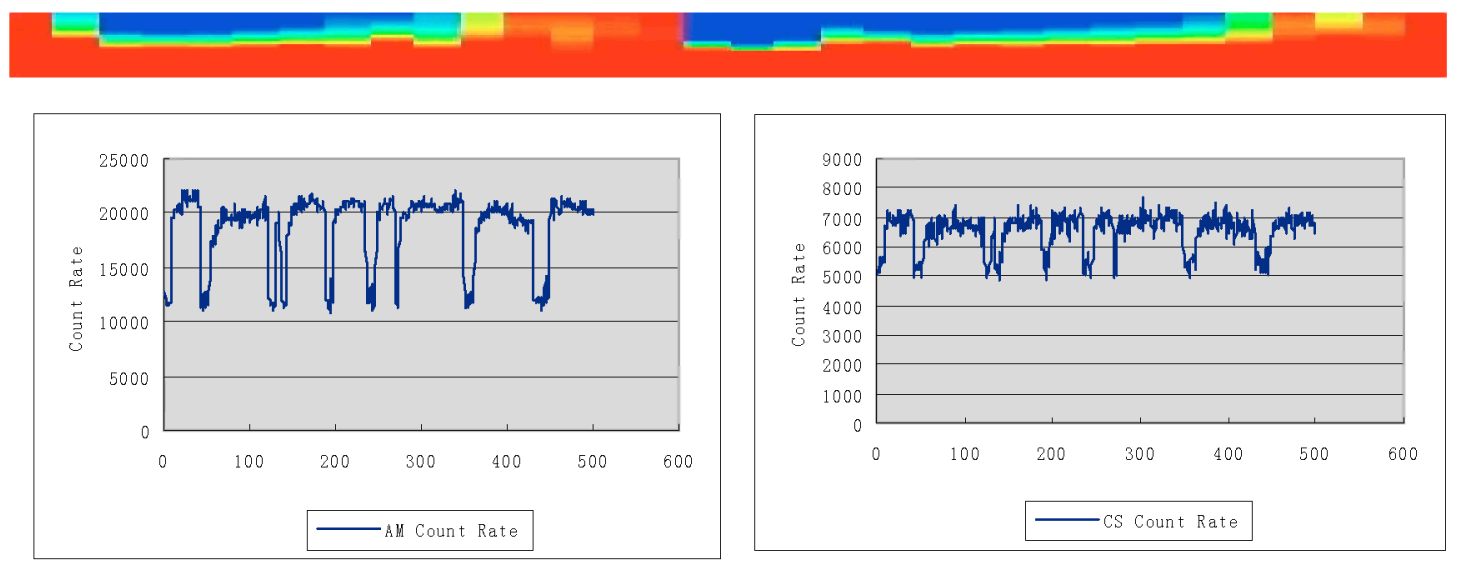

FIGURE 7. Measurements curves

There is statistical fluctuation in $\gamma$ ray source emission intensity. This requires a long integral time in the measurement to reduce the error. The accuracy of both measurement channels is better than $3 \%$ in a single energy measurement when the integral time is longer than 200 seconds. But it becomes worse in a short integral time, for example, the accuracy drops to $1.5 \%$ in $10 \mathrm{~s}$ and even to $5 \%$ in $0.1 \mathrm{~s}$ integrate time. This means that there should be a significant error in dynamic measurement because of the short acquisition time. In fact, during dynamic measurement, due to the moving fluid, in order to obtain the short time flow detail, we must let the $\gamma$ ray system to have high accuracy in short time measurement. The circumstances become even worse in dual energy three phase measurements. In the three phase volumetric fraction measurement, as mentioned in part 2 of this paper, the $\gamma$ ray measurement system relying on photon attenuation in oil and water to distinguish the two materials would obtain a maximum discrimination in the energy region below $40 \mathrm{keV}$, but the lower energy isotopes of ${ }^{241} \mathrm{Am}$ used in this project has the emission energy of $59.5 \mathrm{keV}$, therefore, the linear attenuation coefficients of two energies in oil and water is closer than expected. The too close linear attenuation coefficients may propagate small errors in the measurements of the intensities into large errors according to equations $1 / 2 / 3[6]$, thus a small error in a single energy measurement may be magnified into large error in the dual energy measurement, even up to $100 \%$. The only way to 
solve this problem is to reduce the single energy primary measurement error, using a long integral time (more than $200 \mathrm{~s}$ in this project). A well treated dual energy $\gamma$ ray system has the potential to get the accuracy better than $90 \%$ in average in the oil-water-gas three phase system.

\section{Results for Three Phase Flow}

Six groups of oil-water-gas three phase flow were measured by the dual energy $\gamma$ ray system. The results of three phase volumetric fraction of the oil-water-gas multiphase flow are shown in Fig.8.
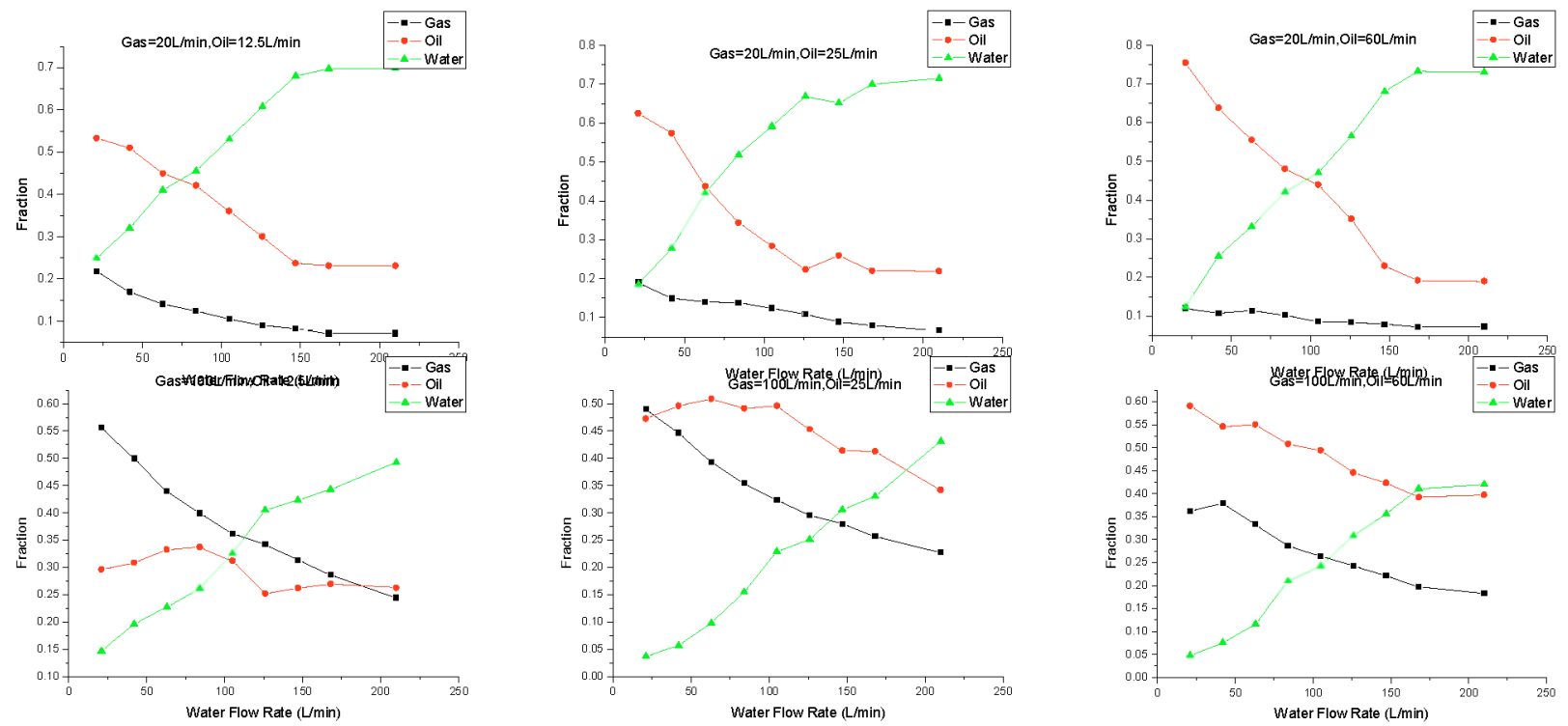

FIGURE 8. Volumetric fractions of oil-water-gas multiphase flow

In the top row of curves in Fig. 8 , the gas flow rate is fixed in $20 \mathrm{~L} / \mathrm{min}$; in bottom row, the gas flow rate is increased to $100 \mathrm{~L} / \mathrm{min}$. The oil flow rate is changed from $12.5 \mathrm{~L} / \mathrm{min}, 25 \mathrm{~L} / \mathrm{min}$ to $60 \mathrm{~L} / \mathrm{min}$ respectively in the diagrams of both rows from left to right in Fig.8. The water flow rate is increased from $21 \mathrm{~L} / \mathrm{min}$ to $210 \mathrm{~L} / \mathrm{min}$ in 9steps as shown on the $\mathrm{X}$ axis in each diagram. The sum of gas, oil and water volumetric fraction is equal to 1 , and the measurement integral time is 200 s in this experiment.

It is easy to find that with the increasing of the oil flow rate as shown in Fig.8 from left to right curves in both rows; the total liquid flow rate increased, and then the gas volumetric fraction decreased. This is because gas is a compressible fluid, when the total liquid fraction increased; the pressure inside the pipe goes up, thus the volume fraction of gas decreased.

The oil volumetric fraction also increased as expected as shown in Fig.8 from left to right curves in both rows. The oil flow rate is changed from $12.5 \mathrm{~L} / \mathrm{min}, 25 \mathrm{~L} / \mathrm{min}$ to $60 \mathrm{~L} / \mathrm{min}$, and the dual energy $\gamma$ ray system gives out a correct response to the fraction changes. This shows a reasonable measurement accuracy. But there are also some significant error points on these curves; some curves do not look smoothly as they should be.

The following diagrams show the oil to water ratio character in experimental measurement related to the oil to water superficial flow rate ratio. The results of oil to water ratio values of oil-water-gas multiphase flow are shown in Fig.9.

In oil-water-gas three phase measurements, it is easier to get better accuracy in gas/liquid measurement. But it is difficult in oil/water discrimination in oil-water-gas three phase. In this group of curves, the black lines of curves are the actual oil to water ratio values, measured by oil and water flow meter, and they are called oil and water superficial flow rate. The red lines of curves are experimental values of oil to water ratio; it is measured by $\gamma$ ray system and obtained by equations $1 / 2 / 3$. 

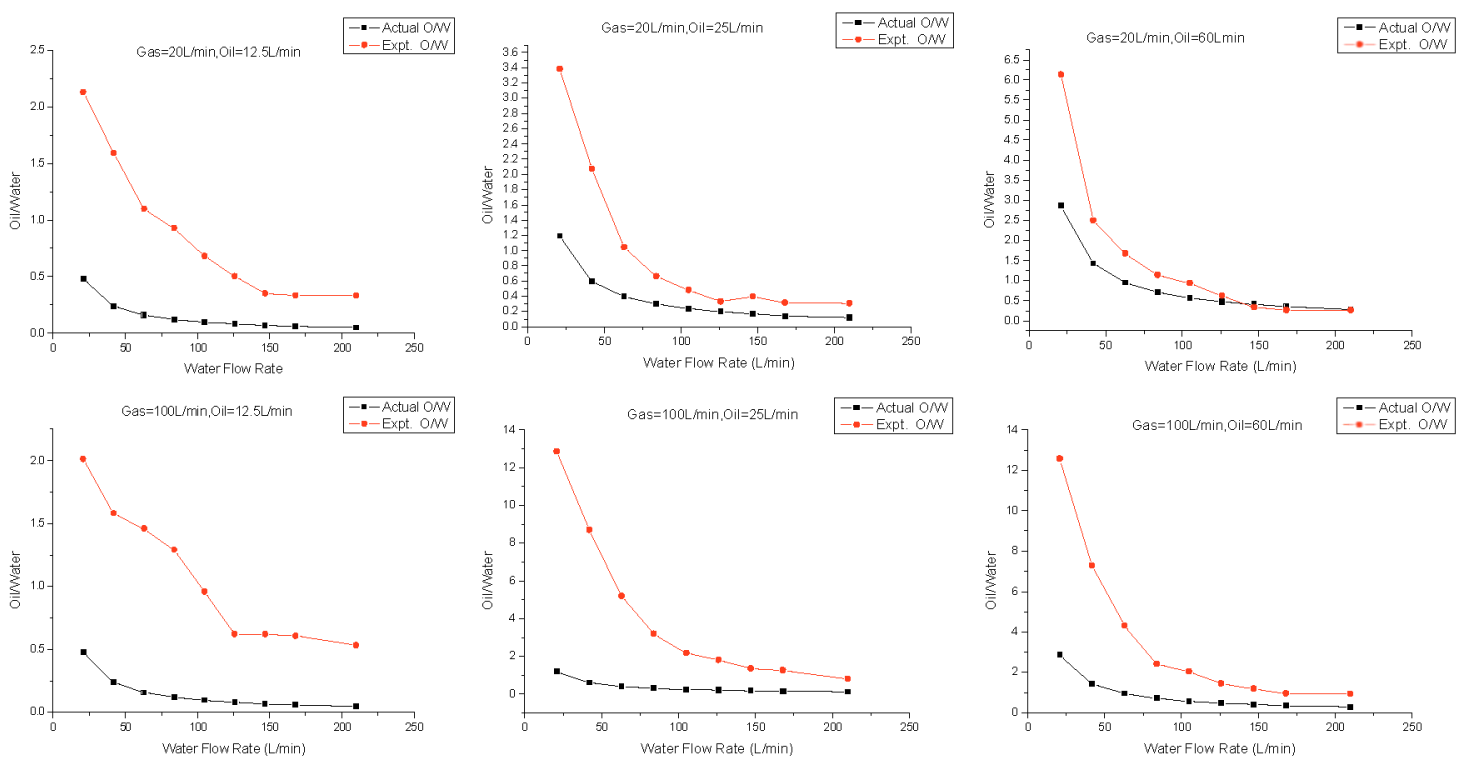

FIGURE 9. Oil to water volumetric fraction of oil-water-gas multiphase flow

From the column 1 in Fig.9, the oil flow rate is only $12.5 \mathrm{~L} / \mathrm{min}$. this is a kind of water dominated flow, and the flow regimes are the stratified flow and long intermittent type flow. It is obvious that the oil to water ratio is much higher than the actual value. This is not a measurement error but it just reflects a phenomenon that the oil speed may be much smaller than water speed in the pipe because oil is more viscous than water, there must be more resistance on the pipe wall to oil especially when oil is less and flow as a dispersed phase in the pipe.

When the oil flow rate is increased to $25 \mathrm{~L} / \mathrm{min}$ even to $60 \mathrm{~L} / \mathrm{min}$, the circumstances are changed. The water's domination becomes less and oil will not flow as dispersed phase. The oil flow resistance begins to influence the water flow speed; this may slow down the water speed, in other hand, the fast water will bring the oil to a faster speed until the oil and water get nearly the same velocity. Then we can find the red curves and the black curves going together in column 2 and column 3 in Fig.9. Meanwhile, the oil and water volumetric fraction get the same with their superficial flow rate ratio.

The other circumstance is the oil dominated flow. This happens in the initial part of the curves in where the water flow rate is only $21 \mathrm{~L} / \mathrm{min}$ or $42 \mathrm{~L} / \mathrm{min}$. In this flow rate of the water, there is no enough energy and enough touch area to push the oil, water only keeps the fast speed itself and leaves the oil behind. This is the reason why there are large differences between the initial parts of two curves in all the diagrams in Fig.9.

The next group of diagrams shows gas to liquid ratio character in the experimental measurement related to the gas and liquid superficial flow rate ratio. The results of gas to liquid ratio values of oil-water-gas multiphase flow are shown in Fig. 10.

In this group of curves, the black lines of curves are the actual gas to liquid ratio values; the liquid flow rate is the total flow rate of water and oil, measured by the oil and water flow meter. The red lines of curves are experimental values of gas to liquid ratio; measured by the $\gamma$ ray system, like the oil to water ratio measurement, the values are obtained by equations $1 / 2 / 3$.

It is easy to find that the red line curves (experimental G/L) are lower than black line curves (actual G/L) in Fig. 10 . This is because the gas is a compressible fluid; the gas flow rate values were measured from the gas mass flow meter, this flow rate is under the standard atmosphere pressure. When the gas enters the pipe, there is a larger pressure inside the pipe, then the gas is compressed in volume, and the gas volumetric fraction at the pipe cross section is reduced as well. So the experimental measured gas to liquid ratio is always lower than the values computed from the superficial flow rate.

In the first row of curves in Fig. 10, the gas flow rate is quite low, only $20 \mathrm{~L} / \mathrm{min}$. The velocity differences between gas and liquid in the pipe are small. Then the red curves and black curves are quite close. But in the second row of curves, the gas flow rate increased to $100 \mathrm{~L} / \mathrm{min}$; t then the gas transport speed increased significantly. The differences between the red curves to black curves become large. 

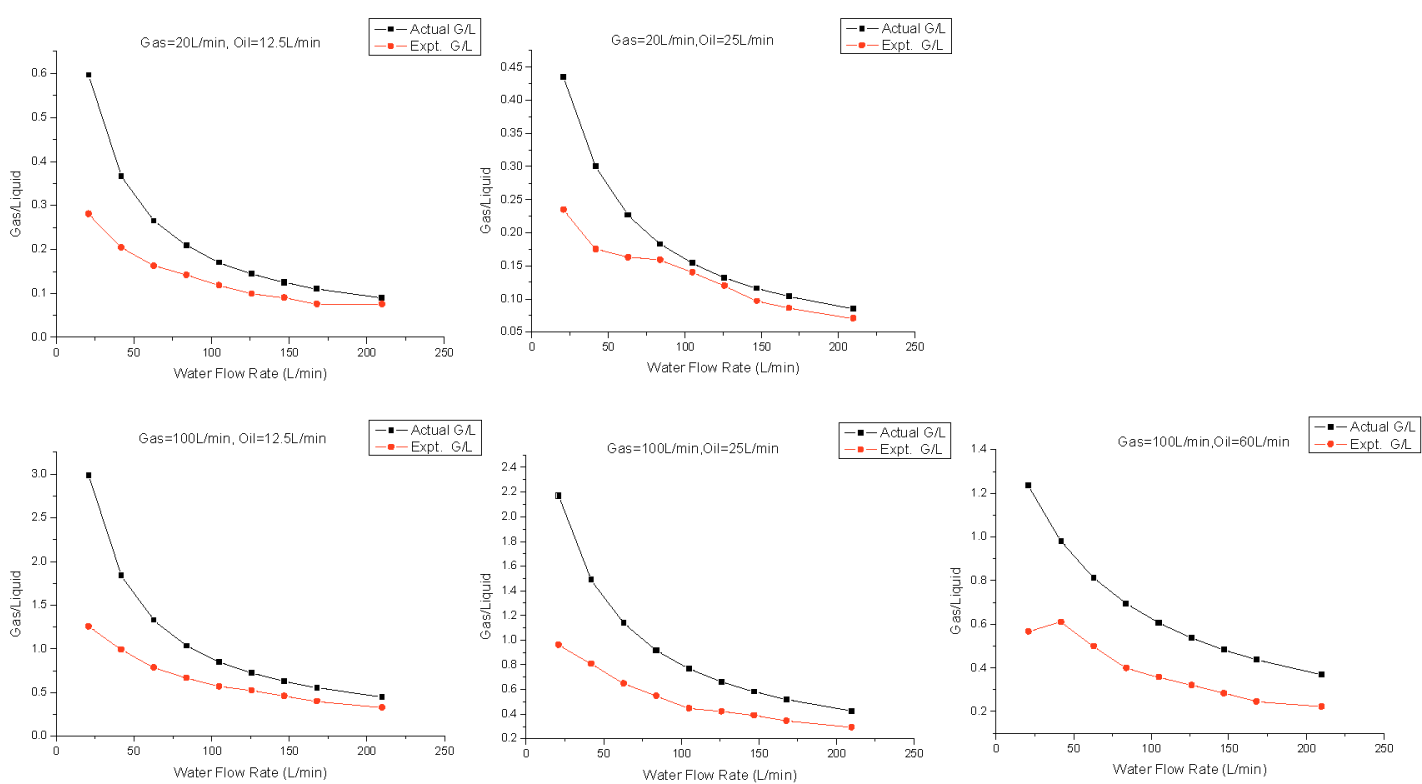

FIGURE 10. Gas to liquid volumetric fraction of oil-water-gas multiphase flow

\section{CONCLUSIONS}

Through the studies on the oil-water-gas three phase horizontal pipe flow with the dual energy $\gamma$ ray system, it is clear that the oil-water-gas three phase flow is very complicated. Not only there are speed shifts and velocity differences between every phase media but also there are phase volumetric fraction changes in the cross section of the pipe. Until now there are no other more efficient and convenient way that can be used to measure the oil-watergas multiphase flow. The dual energy $\gamma$ ray is a promising technique for the purpose of simple and fast estimating the phase fractions of the oil-water-gas multiphase flow.

There is statistical fluctuation in $\gamma$ ray source emission intensity. This requires a long integral time in measurement to reduce the error, and it is difficult to get short time measurement information, only the long time average results are reliable unless uses large activity $\gamma$ ray source, but this will bring the new problems related to system cost and radioactive safety protections.

Dual energy $\gamma$ ray techniques are based on materials attenuation coefficients measurement. In oil-water-gas three phase system, if the lower $\gamma$ ray energy is above $40 \mathrm{keV}\left(59 \mathrm{KeV}^{241} \mathrm{Am}\right.$ in this project), the linear attenuation coefficients of two energies in oil and water is somewhat close to each other. It may propagate small errors in the measurements of the intensities into large errors in the estimation of the thickness through the equation $1 / 2 / 3$. This is another limitation to the measurement accuracy.

Generally, there are still some difficulties in oil-water-gas three phases discrimination using dual energy $\gamma$ ray system, the dynamic results of experimental measurement in this project could not be checked real timely due to the fact that there are no fast switch valves on the flow loop. But the dynamic measurement results and curves are reasonable and repeatable during the experiment..

Finally, a well treated dual energy $\gamma$ ray system has the potential to get the accuracy better than $90 \%$ on averagely in oil-water-gas three phase system.

\section{REFERENCES}

1. M.S.A.Abouelwafa and E.J.M.Kendall, "The Measurement of Component Ratios in Multiphase System Using $\gamma$-ray Attenuation", J. Phy. E: Sci. Instrum. 13, 341(1980)

2. T. Grassler and K.E. Wirth, "Dual-Energy X-Ray Tomography in Process Engineering-A Non-Intrusive Technique to Characterize Vertical Multiphase Flows" 2nd World Congress on Industrial Process Tomography, Hannover, Germany 2001 . 
3. W. Minder and A. Liechti, Röntgenphysik, Springer Verlag, Wien (1955).

4. H. Morneburg (Ed.), Bildgebende Systeme für die medizinische Diagnostik, Publicis MCD Verlag, München(1995)

5. M. J. Key, Gas Microstructure X-Ray Detectors and Tomographic Multiphase Flow Measurement. Ph.D Thesis, University of Surrey(1999).

6. Li Dong-hui, Wu Ying-xiang, Li Zhi-biao, Zhong Xing-fu, "Volumetric Fraction Measurement in Oil-Water-Gas Multiphase Flow with Dual Energy Gamma-Ray System”, J Zhejiang Univ SCI 2005 6A(12):1405-1411. 